\title{
Composición, estructura y diversidad de especies arbóreas en un bosque templado del Noroeste de México
}

\section{Composition, structure and diversity of tree species in a temperate forest in Northwestern Mexico}

\author{
Gabriel Graciano-Ávila ${ }^{1 *}$, Óscar A. Aguirre-Calderón ${ }^{1}$, Eduardo Alanís-Rodríguez ${ }^{1}$, José E. Lujan-Soto ${ }^{1}$ \\ ${ }^{1}$ Facultad de Ciencias Forestales, Universidad Autónoma de Nuevo León. Carretera Linares-Cd. Victoria km 145. Apartado \\ Postal 41. CP. 67700. Linares, Nuevo León, México. \\ *Autor de correspondencia: gabri.e.I@hotmail.com
}

Artículo científico recibido: 27 de mayo de 2016 aceptado: 23 de marzo de 2017

RESUMEN. Se caracterizó la estructura y diversidad de especies arbóreas en un bosque templado del noroeste de

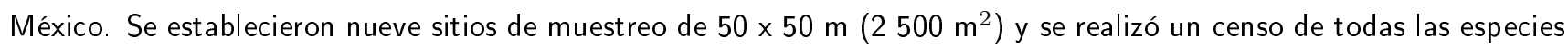
arbóreas. A cada individuo se le realizaron mediciones de altura total y diámetro a la altura del pecho. Se obtuvo el índice de valor de importancia (IVI), calculado a partir de las variables, abundancia, dominancia y frecuencia. También se calcularon los índices diversidad y riqueza. En total se registraron 12 especies, cuatro géneros y cuatro familias. El bosque presenta una densidad de 575.11 individuos ha ${ }^{-1}$ y un área basal de $23.54 \mathrm{~m}^{2} \mathrm{ha}^{-1}$. La especie Pinus cooperi fue la de mayor IVI (79.05\%), y para el índice de Shannon tuvo un valor de 1.74 .

Palabras clave: Bosque templado, Durango, índices de diversidad, Pinus, Quercus

ABSTRACT. The structure and diversity of tree species in a temperate forest in Northwestern Mexico were described.

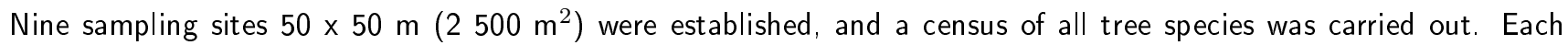
individual was measured: total height and diameter at breast height. The importance value index (IVI) was obtained, based on the following variables: abundance, dominance, and frequency. The diversity and richness indexes were also calculated. Overall, twelve species, four genera, and four families were recorded. The forest has a 575.11 individuals ha ${ }^{-1}$ density and a $23.54 \mathrm{~m}^{2} \mathrm{ha}^{-1}$ basal area. Pinus cooperi was the species that showed the highest IVI (79.05\%), and a Shannon index of 1.74 .

Key words: Temperate forest, Durango, diversity indexes, Pinus, Quercus

\section{INTRODUCCIÓN}

La Sierra Madre Occidental es un complejo montañoso que se extiende desde cerca de la frontera con Estados Unidos de América hasta el norte de Jalisco, recorre más de $1500 \mathrm{~km}$ de norte a sur, representando el $30 \%$ del territorio de México (Descroix 2004). En esta región existen extensas zonas cubiertas por bosques de coníferas y de encinos, lo que da como resultado una flora rica en diversidad de asociaciones de pinos, encinos y madroños (González et al. 2007). De las 52 especies de pino que existen en el país, 20 se encuentran en el estado de Durango (Gernandt y Pérez 2014). La im- portancia de este tipo de vegetación no es sólo por su alta diversidad, sino también porque las especies de pinos y encinos son los árboles de mayor interés económico (Sánchez et al. 2003).

La caracterización estructural es importante para entender el funcionamiento de los ecosistemas, lo cual puede aportar elementos de decisión para contribuir al manejo adecuado de los bosques (Aguirre et al. 2003b, Castellanos-Bolaños et al. 2008). En donde se toman en cuenta índices estructurales y variables dasométricas (Aguirre et al. 2003a). La estructura vertical y horizontal se considera como una buena práctica de manejo forestal para la conservación de la biodiversidad en eco- 


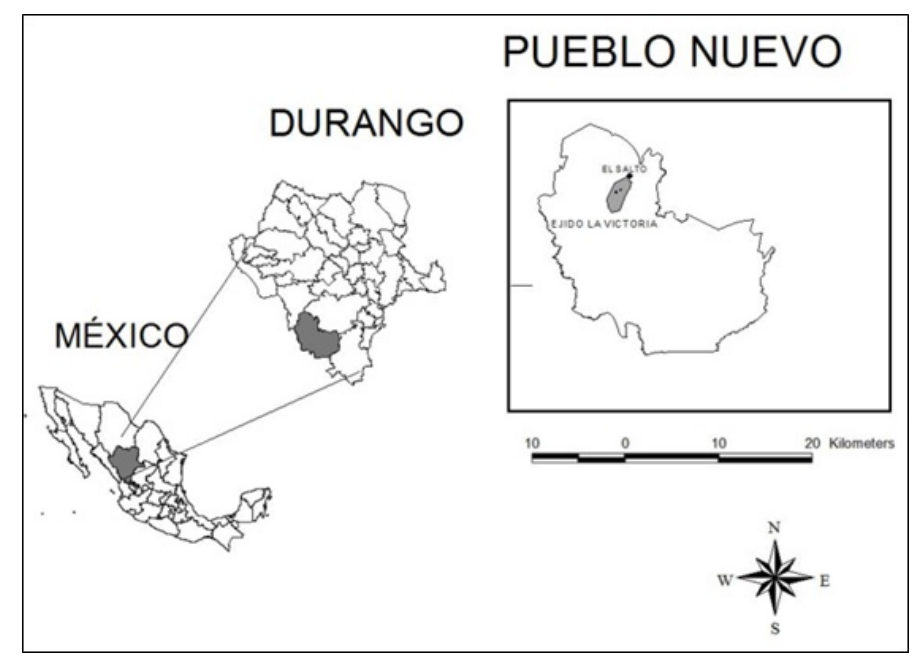

Figura 1. Localización del área de estudio.

sistemas templados (Aguirre-Calderón 2015). La estructura, diversidad y densidad son las principales características de las masas boscosas; la diversidad es un concepto que permite diferentes interpretaciones, aunque en general, se emplea como sinónimo de diversidad de especies (Gadow et al. 2007). Por otro lado la estructura arbórea es un elemento clave para evaluar la estabilidad de los bosques (Lähde et al. 1999), la cual se pude modificar mediante la aplicación de tratamientos silvícolas, cambiando la estructura de los rodales o masas forestales y en consecuencia el bosque (Del Río et al. 2003, Castellanos-Bolaños et al. 2008). En México se han realizado diversos estudios sobre la diversidad de especies arbóreas en clima templado (Návar-Cháidez y González-Elizondo 2009, Leyva et al. 2010). Por lo anterior, el objetivo del presente estudio fue evaluar la composición, estructura y diversidad de especies arbóreas en un bosque templado del noroeste de México.

\section{MATERIALES Y MÉTODOS}

El estudio se realizó en el ejido La Victoria, ubicado al Sudoeste del estado de Durango, en el municipio de Pueblo Nuevo. Enmarcado geográficamente entre los $23^{\circ} 40^{\prime} 04^{\prime \prime}$ y $23^{\circ} 47^{\prime} 54^{\prime \prime}$ LN, y los $105^{\circ} 21^{\prime} 31^{\prime \prime}$ y $105^{\circ} 29^{\prime} 52^{\prime \prime}$ LO (Figura 1 ). Oro- gráficamente, el ejido La Victoria se encuentra ubicado en la provincia fisiográfica de la Sierra Madre Occidental. De acuerdo con la carta edafológica del INEGI (1988), los suelos del área de estudio son tipo cambisol, regosol y litosol con textura gruesa a media. La vegetación está formada por bosques de pino-encino, con distintas condiciones de productividad.

Los datos dasométricos para el estudio se tomaron en nueve parcelas permanentes de muestreo, cuadrangulares de $2500 \mathrm{~m}^{2}$ establecidas en el 2007. La base de datos la constituyeron 1294 árboles a los que se les midió el diámetro normal, la altura total y se registró la especie a la que pertenece cada individuo. Para caracterizar la estructura horizontal se determinó su abundancia, de acuerdo con el número de árboles, su dominancia en función del área basal, y su frecuencia con base en la presencia en los sitios de muestreo. Las variables relativizadas se utilizaron para obtener el índice de valor de importancia (IVI), que adquiere valores porcentuales en una escala de cero a 300 (Mostacedo y Fredericksen 2000), con la fórmula:

$$
A_{i}=\frac{N_{i}}{E} \quad, \quad A R_{i}=\left(\frac{A_{i}}{\sum_{i=1 \ldots n} A_{i}}\right) \times 100
$$




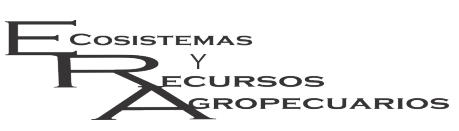

Donde: $\mathrm{A}_{i}$ es la abundancia absoluta, $\mathrm{AR}_{i}$ es la abundancia relativa de la especie $\mathrm{i}$ respecto a la abundancia total, $\mathrm{N}_{i}$ es el número de individuos de la especie i, y E la superficie de muestreo (ha).

La cobertura relativa se obtuvo con la fórmula:

$$
D_{i}=\frac{A b_{i}}{E(h a)} \quad, \quad D R_{i}=\left(\frac{D_{i}}{\sum_{i=1 \ldots n} D_{i}}\right) \times 100
$$

Donde: $\mathrm{D}_{i}$ es la cobertura absoluta, $\mathrm{DR}_{i}$ es cobertura relativa de la especie i respecto a la cobertura, $\mathrm{Ab}$ el área basal de la especie i y $\mathrm{E}$ la superficie (ha). La frecuencia relativa se obtuvo con la fórmula:

$$
F_{i}=\frac{P_{i}}{N S} \quad, \quad F R_{i}=\left(\frac{F_{i}}{\sum_{i=1 \ldots n} F_{i}}\right) \times 100
$$

Donde: $\mathrm{F}_{i}$ es la frecuencia absoluta, $\mathrm{FR}_{i}$ es la frecuencia relativa de la especie i respecto a la suma de las frecuencias, $\mathrm{P}_{i}$ es el número de sitios en el que está presente la especie i y NS el número total de sitios de muestreo.

El índice de valor de importancia (IVI) se define como (Whittaker 1972, Moreno 2001):

$$
I V I=A R_{i}+D R_{i}+F R_{i}
$$

Para estimar la riqueza de especies se utilizó el índice de Margalef $\left(\mathrm{D}_{M g}\right)$ y para la diversidad alfa el índice de Shannon-Weaver $\left(\mathrm{H}^{\prime}\right)$ mediante las fórmulas (Shannon 1948, Magurran 2004):

$D_{m g}=\frac{(S-1)}{\ln (N)}, H^{\prime}=-\sum_{i=1}^{S} p_{i} \times \ln \left(p_{i}\right), P_{i}=\frac{n_{i}}{N}$

Donde: $\mathrm{S}$ es el número de especies presentes, $\mathrm{N}$ es el número total de individuos, $\mathrm{n}_{i}$ es el número de individuos de la especie $\mathrm{i}$ y $\mathrm{p}_{i}$ es la proporción de individuos de la especie i respecto al total de individuos. Para la caracterización de la estructura vertical de las especies se utilizó el índice de distribución vertical de especies (A) (Del Río et al. 2003). Donde A tiene valores entre cero y un valor
Caracterización de un bosque en México

Ecosist. Recur. Agropec.

4(12):535-542,2017

máximo $\left(\mathrm{A}_{\max }\right)$; cuando un valor $\mathrm{A}=0$ significa que el rodal está constituido por una sola especie que ocurre en un solo estrato. $A_{\max }$ se alcanza cuando la totalidad de las especies ocurren en la misma proporción tanto en el rodal como en los diferentes estratos (Corral et al. 2005). Para la estimación de distribución vertical de las especies, se definieron las zonas de altura (Jiménez 2001): zona I: 80 a $100 \%$ de la altura máxima del área; zona II: 50 a $80 \%$ de la altura máxima, y zona III: de cero a $50 \%$ de la altura máxima. El índice se estimó con la siguiente fórmula:

$$
A=-\sum_{i=1}^{S} \sum_{j=1}^{Z} p_{i j}^{*} \ln \left(p_{i j}\right), A \max =\ln (S * Z)
$$

Donde: $S=$ número de especies presentes; $Z=$ número de estratos de altura; $\mathrm{p}_{i j}=$ porcentaje de especies en cada zona, y se estima mediante la siguiente ecuación $\mathrm{p}_{i j}=\mathrm{n}_{i, j} / \mathrm{N}$; donde $\mathrm{n}_{i, j}=$ número de individuos de la misma especie (i) en la zona (j) y $\mathrm{N}=$ número total de individuos. El valor de $\mathrm{A}$ se estandariza de la siguiente forma:

$$
A_{\text {rel }}=\frac{A}{\ln (S * Z)} * 100
$$

\section{RESULTADOS}

Se registraron 12 especies arbóreas en total, distribuidas en cuatro géneros y cuatro familias (Tabla 1). La familia más representativa fue Pinaceae con cinco especies, seguida de las familias Fagaceae y Ericaceae con tres especies cada una. Estas tres familias incluyeron tres géneros y 11 especies, lo que constituye el $91.7 \%$ de la vegetación registrada en los nueve sitios de muestreo.

La comunidad vegetal del área de estudio tuvo una densidad de 575.11 individuos ha ${ }^{-1}$. El género Pinus fue el más abundante con densidad de 369.89 árboles ha ${ }^{-1}$, representando el $69.01 \%$ del total, seguido del género Quercus que presentó una densidad de 108 árboles ha ${ }^{-1}$ con el $18.78 \%$ del total (Tabla 2). Las especies con mayor densidad fueron Pinus cooperi (188.89 árboles ha ${ }^{-1}$ ) con 
Tabla 1. Nombre científico y familia de especies arbóreas registradas en el área de estudio.

\begin{tabular}{ll}
\hline Nombre científico & Familia \\
\hline Arbutus bicolor S. González & Ericaceae \\
Arbutus madrensis S. González & Ericaceae \\
Arbutus xalapensis Kunth & Ericaceae \\
Juniperus deppeana Steud. & Cupressaceae \\
Pinus ayacahuite Ehrenb. ex Schltdl. & Pinaceae \\
Pinus cooperi C.E.Blanco & Pinaceae \\
Pinus durangensis Martínez & Pinaceae \\
Pinus leiophylla Schiede ex Schltdl. \& Cham. & Pinaceae \\
Pinus teocote Schied. ex Schltdl. \& Cham. & Pinaceae \\
Quercus crassifolia Bonpl. & Fagaceae \\
Quercus rugosa Née & Fagaceae \\
Quercus sideroxyla Bonpl. & Fagaceae \\
\hline
\end{tabular}

Tabla 2. Abundancia, dominancia y frecuencia por género de las especies registradas en el área de estudio.

\begin{tabular}{lrrrrrr}
\hline \multirow{2}{*}{ Género } & \multicolumn{2}{c}{ Abundancia } & \multicolumn{2}{c}{ Dominancia } & \multicolumn{2}{c}{ Frecuencia } \\
\cline { 2 - 7 } & \multicolumn{1}{c}{ Absoluta } & \multicolumn{1}{c}{ Relativa } & \multicolumn{1}{c}{ Absoluta } & Relativa & Absoluta & Relativa \\
\cline { 2 - 7 } & $\left(\mathrm{n} \mathrm{ha}^{-1}\right)$ & \multicolumn{1}{c}{$(\%)$} & $\left(\mathrm{m}^{2} \mathrm{ha}^{-1}\right)$ & \multicolumn{1}{c}{$(\%)$} & \multicolumn{1}{c}{-} & $(\% 0)$ \\
\hline Pinus & 396.89 & 69.01 & 16.71 & 70.98 & 100.00 & 30.00 \\
Quercus & 108.00 & 18.78 & 5.58 & 23.71 & 77.78 & 23.33 \\
Juniperus & 52.89 & 9.20 & 0.74 & 3.15 & 88.89 & 26.67 \\
Arbutus & 17.33 & 3.01 & 0.51 & 2.16 & 66.67 & 20.00 \\
Total & 575.11 & 100.00 & 23.54 & 100.00 & 333.33 & 100.00 \\
\hline
\end{tabular}

$32.84 \%$ del total, $P$. durangensis (143.56 árboles $\mathrm{ha}^{-1}$ ) con $24.94 \%$ del total, Quercus sideroxila (104.44 árboles ha ${ }^{-1}$ ) con $18.16 \%$ del total y $J u$ niperus deppeana (52.89 árboles ha ${ }^{-1}$ ) con $9.2 \%$ del total, lo cual equivale al $85.16 \%$ del total de especies. Mientras que la especie menos abundante fue $Q$. rugosa ( 1 árbol ha ${ }^{-1}$ ) con $0.08 \%$ del total (Tabla 3).

Se tuvo una dominancia de área basal de $23.54 \mathrm{~m}^{2} \mathrm{ha}^{-1}$. El género Pinus fue el de mayor dominancia relativa $\left(16.71 \mathrm{~m}^{2} \mathrm{ha}^{-1}\right)$ con $70.98 \%$ del total, seguido del género Quercus que tuvo una dominancia relativa de $5.58 \mathrm{~m}^{2} \mathrm{ha}^{-1}$, lo que equivale al $23.71 \%$ del total (Tabla 2). Las especies con mayor área basal fueron $P$. durangensis $\left(7.76 \mathrm{~m}^{2} \mathrm{ha}^{-1}\right)$ con $32.97 \%$, P. cooperi $\left(6.96 \mathrm{~m}^{2}\right.$ $\mathrm{ha}^{-1}$ ) con $29.55 \%$, Q. sideroxila (3.63 $\mathrm{m} 2 \mathrm{ha}^{-1}$ ) con $15.40 \%$ y $Q$. crassifolia $\left(1.95 \mathrm{~m}^{2} \mathrm{ha}^{-1}\right)$ con $8.29 \%$, que en total suman el $86.22 \%$ del total de especies.

El género Pinus se presentó en todos los sitios de muestreo, seguido del genero Juniperus que se encontró en ocho de los nueve sitios de muestreo (Tabla 2). A nivel especie, $P$. cooperi se encontró en los nueve sitios de muestreo (100\% de frecuencia absoluta) con $16.67 \%$ de frecuencia relativa, seguido de J. deppeana que se encontró en ocho sitios de muestreo con frecuencia absoluta del 88 $\%$, lo que representa el $14.81 \%$ de frecuencia relativa. Las especies Arbutus xalapensis, A. madrensis, $Q$. rugosa y $Q$. crassifolia sólo se presentaron en un sitio de muestreo (Tabla 3).

El índice de valor de importancia indica que P. cooperi $(79.05 \%)$, P. durangensis $(70.89 \%)$, Q. sideroxyla $(46.53 \%)$ y J. deppeana $(27.18 \%)$ fueron las especies más sobresalientes, al tener los mayores valores de IVI. Las especies más raras fueron $A$. xalapensis, $A$. madrensis y $Q$. rugosa que tuvieron valores menores del tres por ciento.

La riqueza específica de la comunidad vegetal estudiada fue de 12 especies, con un índice de Margalef de 1.53. En relación al valor de diversidad de especies, el valor del índice de Shannon fue de 1.74. Por el índice de distribución vertical de las especies se definieron tres estratos de altura, alto (31.04 $38.80 \mathrm{~m})$, medio $(19.40-31.03 \mathrm{~m})$ y bajo $(<19$ - $40 \mathrm{~m}$ ). El estrato alto se encuentra conformado por $P$. cooperi, $P$. durangensis y $P$. teocote con 
Tabla 3. Parámetros estructurales estimados para las especies registradas en el área de estudio. IVI = Índice de Valor de Importancia. Las especies están ordenadas en forma descendente según su IVI.

\begin{tabular}{lrrrrrrr}
\hline \multirow{2}{*}{ Género } & \multicolumn{2}{c}{ Abundancia } & \multicolumn{2}{c}{ Dominancia } & \multicolumn{2}{c}{ Frecuencia } & \\
\cline { 2 - 6 } & Absoluta & Relativa & \multicolumn{1}{c}{ Absoluta } & Relativa & Absoluta & Relativa & IVI \\
\cline { 2 - 6 } & $\left(\mathrm{n} \mathrm{ha}^{-1}\right)$ & \multicolumn{1}{c}{$(\%)$} & $\left(\mathrm{m}^{2} \mathrm{ha}^{-1}\right)$ & \multicolumn{1}{c}{$(\%)$} & - & \multicolumn{1}{c}{$\%)$} & \\
\hline Pinus cooperi & 188.89 & 32.84 & 6.96 & 29.55 & 100.00 & 16.67 & 79.05 \\
Pinus durangensis & 143.56 & 24.96 & 7.76 & 32.97 & 77.78 & 12.96 & 70.89 \\
Quercus sideroxyla & 104.44 & 18.16 & 3.62 & 15.40 & 77.78 & 12.96 & 46.53 \\
Juniperus deppeana & 52.89 & 9.20 & 0.74 & 3.15 & 88.89 & 14.81 & 27.18 \\
Pinus ayacahuite & 32.00 & 5.56 & 1.05 & 4.44 & 77.78 & 12.96 & 22.98 \\
Pinus teocote & 27.11 & 4.71 & 0.85 & 3.60 & 66.67 & 11.11 & 19.41 \\
Arbutus bicolor & 14.22 & 2.47 & 0.32 & 1.38 & 44.44 & 7.41 & 11.25 \\
Quercus crassifolia & 3.11 & 0.54 & 1.95 & 8.29 & 11.11 & 1.85 & 10.68 \\
Pinus leiophylla & 5.33 & 0.93 & 0.10 & 0.42 & 22.22 & 3.70 & 5.04 \\
Arbutus xalapensis & 2.22 & 0.39 & 0.16 & 0.70 & 11.11 & 1.85 & 2.94 \\
Arbutus madrensis & 0.89 & 0.15 & 0.02 & 0.08 & 11.11 & 1.85 & 2.1 \\
Quercus rugosa & 0.44 & 0.08 & 0.00 & 0.01 & 11.11 & 1.85 & 1.95 \\
Total & 575.11 & 100.00 & 23.54 & 100.00 & 600.00 & 100.00 & 300 \\
\hline
\end{tabular}

Tabla 4. Valores del índice vertical de Pretzch para el área de estudio.

\begin{tabular}{lrrrr}
\hline & \multicolumn{4}{c}{ Proporción (\%) } \\
\hline Estrato alto(38.80 - 31.04) & $\mathrm{N}$ ha ${ }^{-1}$ & Del total & En la zona \\
Pinus cooperi & 3.00 & 1.33 & 30.00 & 0.23 \\
Pinus durangensis & 6.00 & 2.67 & 60.00 & 0.46 \\
Pinus teocote & 1.00 & 0.44 & 10.00 & 0.08 \\
Suma & 10.00 & 4.44 & 100.00 & 0.77 \\
& & & & \\
Estrato medio(31.04 - 19.40) & & & & \\
Pinus ayacahuite & 67.00 & 29.78 & 34.01 & 5.18 \\
Pinus cooperi & 33.00 & 14.67 & 16.75 & 2.55 \\
Pinus durangensis & 81.00 & 36.00 & 41.12 & 6.26 \\
Pinus teocote & 4.00 & 1.78 & 2.03 & 0.31 \\
Quercus crassifolia & 3.00 & 1.33 & 1.52 & 0.23 \\
Quercus sideroxyla & 9.00 & 4.00 & 4.57 & 0.70 \\
Suma & 197.00 & 87.56 & 100.00 & 15.22 \\
& & & & \\
Estrato bajo(2.50 - 19.4) & & & & \\
Arbutus bicolor & 32.00 & 14.22 & 2.94 & 2.47 \\
Arbutus madrensis & 2.00 & 0.89 & 0.18 & 0.15 \\
Arbutus xalapensis & 5.00 & 2.22 & 0.46 & 0.39 \\
Juniperus deppeana & 119.00 & 52.89 & 10.95 & 9.20 \\
Pinus ayacahuite & 5.00 & 2.22 & 0.46 & 0.39 \\
Pinus cooperi & 389.00 & 172.89 & 35.79 & 30.06 \\
Pinus durangensis & 236.00 & 104.89 & 21.71 & 18.24 \\
Pinus leiophylla & 12.00 & 5.33 & 1.10 & 0.93 \\
Pinus teocote & 56.00 & 24.89 & 5.15 & 4.33 \\
Quercus crassifolia & 4.00 & 1.78 & 0.37 & 0.31 \\
Quercus sideroxyla & 226.00 & 100.44 & 20.79 & 17.47 \\
Quercus rugosa & 1.00 & 0.44 & 0.09 & 0.08 \\
Suma & 1087.00 & 483.11 & 100.00 & 84.00 \\
Total & 1294.00 & 575.11 & 300.00 & 100.00 \\
\hline
\end{tabular}

4.44 árboles $\mathrm{ha}^{-1}$, lo que equivale al $0.77 \%$ de la zona. El estrato medio lo conforman $P$. ayacahuite, $P$. cooperi, $P$. durangensis, $P$. teocote, $Q$. crassifolia y $Q$. sideroxyla con 87.56 árboles ha ${ }^{-1}$ que representan el $15.22 \%$ de la zona. En el estrato bajo se registraron todas las especies del área de estudio con 483.11 árboles ha ${ }^{-1}$ lo que representa el $84 \%$ de la zona. La especie más abundante fue $P$. cooperi con 172.89 árboles ha ${ }^{-1}$ seguida de $P$. durangensis con 104.89 árboles ha ${ }^{-1}$ y $Q$. sideroxyla con 100.44 árboles ha ${ }^{-1}$ (Tabla 4). El valor del índice $A$ fue de 2.07 con un valor de $\mathrm{A}_{\max }$ de 3.58 y $\mathrm{A}_{r e l}$ de $57 \%$, 
lo que indica que la zona evaluada tiene uniformidad media, en diversidad de alturas. Los valores de Arel cercanos a $100 \%$ indican que todas las especies se encuentran distribuidas de forma equitativa en los tres estratos de altura.

\section{DISCUSIÓN}

Por su estructura y composición, el área de estudio evaluada corresponde a un bosque típico del macizo montañoso de la Sierra Madre Occidental (González et al. 2007). Se encontraron las mismas familias dominates reportadas para la Sierra Madre Occidental del estado de Durango, las cuales son Pinaceae y Fagaceae (Aragón-Piña et al. 2010). La familia Pinaceae fue la más abundante al registrar cinco especies del género Pinus, lo que coincide con García y González (1998), quienes señalan que la distribución de la familia Pinaceae y el género Pinus es amplia en todas las cadenas montañosas del país. Estos resultados coinciden con lo registrado para el estado de en Chihuahua, donde se reportan como dominantes a las especies del genero Pinus (Hernández-Salas et al. 2013). Mientras que López et al. (2017) encontraron que el género Pinus también tiene mayor abundancia en los bosques templados. Se registraron 12 especies, cantidad inferior a las 27 especies registradas por Linares et al. (1999). La especie P. cooperi fue la más abundante, la cual es una de las especies reportadas como de las más abundante en los bosques templados (Návar-Cháidez y González-Elizondo 2009, Valenzuela y Granados 2009). Las especies con mayor importancia por el área basal que ocupan $86.22 \%$ fueron $P$. durangensis, $P$. cooperi, $Q$. sideroxyla y $Q$. crassifolia; estas mismas especies han sido reportadas como las de mayor área basal en bosques de pino encino del estado de Durango con valores superiores al 65 \% (Valenzuela y Granados 2009). Las dos especies con el índice de valor de importancia más alto fueron $P$. cooperi y $P$. durangensis con el 26.35 y $23.63 \%$, respectivamente; al respecto $P$. durangensis ha sido reportada como la especie con el mayor valor ecológico (57.05 $\%$ ) en bosques templados (Hernández-Salas et al.
2013). Sobre lo mismo González et al. (1993) reportan que la especie $P$. cooperi es la de mayor valor de importancia ecológica (19.92\%); mientras que Valenzuela y Granados (2009) reportan a P. durangensis y $P$. cooperi como las especies con mayor valor de importancia ecología con el 33.44 y $10.0 \%$, respectivamente. Al respecto Margalef (1972) menciona que el índice de Shannon normalmente varía de 1 a 5 , interpretándose los valores menores de 2 como diversidad baja, de 2 a 3.5 diversidad media, y mayores de 3.5 como diversidad alta. Por lo que la comunidad forestal estudiada presenta una diversidad baja $\left(H^{\prime}=1.74\right)$. Pero este valor es superior al registrado por Návar-Cháidez y González-Elizondo (2009) para los bosques templados del estado de Durango. El valor del índice de Margalef $\left(\mathrm{D}_{M g}=\right.$ 1.53) es superior a los reportados por HernándezSalas et al. (2013), quienes registraron valores de $\mathrm{D}_{M g}=1.04$ y $\mathrm{D}_{M g}=0.90$ respectivamente. Lo que significa que el lugar de estudio presenta mayor diversidad de especies arbóreas si es comparado con áreas de la misma región, sin embargo, este valor es bajo si se compara con los valores reportados para otros ecosistemas (Alanís et al. 2008, Agis et al. 2016). El índice A tuvo valores de 2.07 con valor $A_{\text {max }}$ de 3.58 y $A_{\text {rel }}$ de $57 \%$, lo que indica que la zona evaluada presenta uniformidad media, en la diversidad de alturas. Valores de $\mathrm{A}_{\text {rel }}$ cercanos a $100 \%$ indican que todas las especies se encuentran distribuidas de forma equitativa en los tres estratos de altura. Lo que coincide con lo reportado por Camacho et al. (2014).

\section{CONCLUSIONES}

El bosque templado estudiado presenta una baja riqueza específica y diversidad de especies arbóreas. Las familias con mayor importancia por su contribución estructural a este bosque son Pinaceae y Fabaceae, los géneros Pinus y Quercus son los más importantes. Mientras que las especies más importantes son $P$. cooperi, $P$. durangensis y $Q$. sideroxyla. Para la estructura vertical, la diversidad de alturas es media por lo que la etapa de desarrollo es latizal. 


\section{LITERATURA CITADA}

Agis J, Castro NDH, Martínez JL, Umaña MR (2016) Diversidad y estructura de la selva mediana subperennifolia de Acapulco, Gro., México. Revista Iberoamericana de las Ciencias Biológicas y Agropecuarias 5: $1-20$.

Aguirre COA, Jiménez Pérez J, Kramer H, Akça A (2003a) Análisis estructural de ecosistemas forestales en el Cerro del Potosí, Nuevo León, México. Ciencia UANL 6: 219-225.

Aguirre O, Hui GY, Gadow KV, Jiménez J (2003b) An analysis of spatial forest structure using neighbourhoodbased variables. Forest Ecology and Management 183: 137-145.

Aguirre-Calderón OA (2015) Manejo Forestal en el Siglo XXI. Madera y Bosques 21: 17-28.

Alanís E, Jiménez J, Aguirre OA, Treviño EJ, Jurado E, González M (2008) Efecto del uso del suelo en la fitodiversidad del matorral espinoso tamaulipeco. Ciencia UANL 11: 56-62.

Aragón-Piña EE, Garza-Herrera A, González-Elizondo MS, Luna-Vega I (2010) Composición y estructura de las comunidades vegetales del rancho El Durangueño, en la Sierra Madre Occidental, Durango, México. Revista Mexicana de Biodiversidad 81: 771-787.

Camacho R, Alonso E, González-Tagle MA, Jiménez-Pérez J, Alanís-Rodríguez E, Ávila-Flores DY (2014) Diversidad y distribución vertical de especies vegetales mediante el índice de Pretzsch. Ciencia UANL 17: 34-41.

Castellanos-Bolaños JF, Treviño-Garza EJ, Aguirre-Calderón ÓA, Jiménez-Pérez J, Musalem-Santiago M, López-Aguillón R (2008) Estructura de bosques de Pino patula bajo manejo en Ixtlán de Juárez, Oaxaca, México. Madera y Bosques 14: 51-63.

Corral J, Aguirre OA, Jiménez J, Corral S (2005) Un análisis del efecto del aprovechamiento forestal sobre la diversidad estructural en el Bosque Mesófilo de Montaña "El Cielo", Tamaulipas, México. Investigaciones Agrarias: Sistema de Recursos Forestales 14: 217-228.

Del Río M, Montes F, Cañellas I y Montero G (2003) Índices de diversidad estructural en masas forestales. Investigaciones Agrarias: Sistema de Recursos Forestales 12: 159-176.

Descroix L, González Barrios JL, Estrada Ávalos (2004) La Sierra Madre Occidental, una fuente de agua amenazada. Instituto Nacional de Investigaciones Forestales, Agrícolas y Pecuarias-Institut de Recherche pour le Développement. Gómez Palacio, Durango, México. 300p.

Gadow KV, Sánchez OS, Álvarez JG (2007) Estructura y crecimiento del bosque. Universidad de Göetingen, Alemania. 287p.

García AA y González EMS (1998) Pináceas de Durango. CIIDIR-IPN, Instituto de Ecología A.C., SIVILLAGobierno del Estado de Durango. Durango, Dgo. México. 179p.

Gernandt DS, Pérez JA (2014) Biodiversidad de Pinophyta (coníferas) en México. Revista Mexicana de Biodiversidad 85: 126-133.

González E, González M, Márquez M (2007) Vegetación y ecorregiones de Durango. Instituto Politécnico Nacional. México. 219p.

González S, González M, Cortés A (1993) Vegetación de la Reserva de la Biosfera "La Michilía", Durango, México. Acta Botánica Mexicana 22: 1-104. 
Hernández-Salas J, Aguirre-Calderón ÓA, Alanís-Rodríguez E, Jiménez-Pérez J, Treviño-Garza EJ, GonzálezTagle MA, et al. (2013) Efecto del manejo forestal en la diversidad y composición arbórea de un bosque templado del noroeste de México. Revista Chapingo. Serie Ciencias Forestales y del Ambiente 19: 189-200.

INEGI (1988) Carta topográfica. Escala 1:50,000. El Salto, Durango. F13- A18 spp. México. http://internet. contenidos.inegi.org.mx/contenidos/Productos/prod_serv/contenidos/espanol/bvinegi/productos/geografia/historia/1_50_000/702825617394.pdf. Fecha de consulta 15 de junio de 2017.

Jiménez J, Aguirre O, Kramer H (2001) Análisis de la estructura horizontal y vertical en un ecosistema multicohortal de pino-encino en el norte de México. Investigaciones Agrarias: Sistema de Recursos Forestales 10: 355-366.

Lähde E, Laiho O, Norokorpi Y, Saksa T (1999) Stand structure as the basis of diversity index. Forest Ecology and Management 115: 213-220.

Leyva JC, Velazquez A, Angeles G (2010) Patrones de diversidad de la regeneración natural en rodales mezclados de pino. Revista Chapingo. Serie Ciencias Forestales y del Ambiente 16: 227-239.

Linares MAM, Elizondo SG, Zagoya RA (1999) Componentes de la diversidad arbórea en bosques de pino encino de Durango, México. Madera y Bosques 5: 67-78.

López JA, Aguirre-Calderón OA, Alanís-Rodríguez E, Monarrez JC, González-Tagle MA, Jiménez-Pérez J (2017) Composición y diversidad de especies forestales en bosques templados de puebla, México. Madera y Bosques 23: 39-51.

Magurran AE (2004) Measuring biological diversity. Blackwell. Cambridge, USA. 256p.

Margalef R (1972) Homage to E. Hutchison, or why is there an upper limit to diversity. Transactions of the Connecticut Academy of Arts and Sciences 44: 211-235.

Moreno CE (2001) Métodos para medir la biodiversidad. Manual y tesis SEA. Editado por Cooperación Iberoamericana, Unesco y SEA. Vol. 1. Pachuca, Hidalgo, México. 83p.

Mostacedo B, Fredericksen S (2000) Manual de métodos básicos de muestreo y análisis en ecología vegetal. BOLFOR; Santa Cruz, Bolivia. 87p.

Návar-Cháidez JDJ, González-Elizondo S (2009) Diversidad, estructura y productividad de bosques templados de Durango, México. Polibotánica 27: 71-87.

Sánchez O, Vega E, Peters E, Monrroy VO (2003) Conservación de ecosistemas templados de montaña en México. Instituto Nacional de Ecología-SEMARNAT. México. 315p.

Shannon C (1948) The mathematical theory of communication. En: The mathematical theory of communication. In: Shannon CE, Weaver W (Ed). University of Illinois. Press Urbana. Illinois, USA. pp: 29-125.

Valenzuela NLM, Granados SD. (2009) Caracterización fisonómica y ordenación de la vegetación en el área de influencia de El Salto, Durango, México. Revista Chapingo. Serie Ciencias Forestales y del Ambiente 15: $29-41$.

Whittaker RH (1972) Evolution and measurement of species diversity. Taxon 21: 213-251. 